Natural Hazards and Earth System Sciences (2003) 3: 255-262

(C) European Geosciences Union 2003

\title{
Distribution of neutrons near the Earth's surface
}

\author{
B. M. Kuzhevskij, O. Yu. Nechaev, and E. A. Sigaeva \\ Skobeltsyn Institute of Nuclear Physics, Lomonosov Moscow State University, Vorobjovy Gory, Moscow, 119899, Russia
}

Received: 30 May 2002 - Revised: 9 October 2002 - Accepted: 28 October 2002

\begin{abstract}
The distribution of the count rate of neutrons (per second) near the Earth's surface for two directions: towards the Earth and away from it, is studied using the experimental data, obtained in Moscow during 1996. The analysis shows that the mathematical approximation of the neutron count rate distribution can be described by a sum of two functions: a Poison distribution and a log-normal distribution. This is in agreement with the two known sources of the total neutron flux near the Earth's surface: generation of neutrons in nuclear interactions of high-energy cosmic ray particles with the Earth's atmosphere and neutron production in the Earth's crust. The log-normal distribution describes the contribution of the Earth's crust to the total neutron flux near the Earth. Therefore, these dynamic processes in the Earth's crust change the parameters of the log-normal distribution.
\end{abstract}

\section{Introduction}

Composition, time variations and spatial distribution of nuclear radiation particles have been studied in the lower atmosphere for about 50 years. In the present work we study only the neutral component (neutrons) of nuclear irradiation.

In previous works scientists did not distinguish between the different sources of neutrons and their contribution to the total neutron flux near the Earth's crust. Many scientists studied the energy spectrum and the altitude, latitude and longitude distributions of the neutron flux in the atmosphere (Gorshkov et al., 1966). The results of these studies lay within the following frames: the neutron component of nuclear radiation in the atmosphere is produced in interactions between high-energy primary cosmic ray particles and nuclei of the atmospheric elements. It was also revealed that in the lower atmosphere the neutron flux is characterized by a very weak latitudinal dependence and an unusual altitude dependence. In one of his experiments, Yuan (Yuan, 1948) obtained that

Correspondence to: B. M. Kuzhevskij

(bmk@srd.sinp.msu.ru) the neutron flux decreases (with increasing altitudes), up to an altitude of several hundreds meters and then increases.

In earlier studies (Kuzhevskij et al., 1995, 1996), we reported that up to altitudes of 1-2 km from the Earth's surface the neutron count rate has no altitude dependence. We called this phenomenon "the neutron field of the Earth". The time distribution of the neutron flux in the neutron field of the Earth can be described as:

$F(t)=n(t) v$,

where $n(t)$ is the time distribution of neutron concentration in the neutron field of the Earth, $v$ is the velocity of neutrons.

Experimental studies of the energetic spectrum of neutrons near the Earth's crust (Beliaeva, 1999) have shown that for over $70 \%$ of the neutrons the energy does not exceed $0.5 \mathrm{eV}$. Theoretical consideration of propagation of neutrons with primary energy of about $1 \mathrm{MeV}$ have shown, that during a period of less than $1 \mathrm{~s}$ (its actual value is about $0.1 \mathrm{~s}$ ), they are thermalized and during a period less than $0.1 \mathrm{~s}$, they are captured by atmospheric nitrogen (Kuzhevskij, 2000). As a result, the neutron velocity in Eq. (1), $v$, is some mean velocity, and the time variation $F(t)$, as we mentioned above, will be defined only by the time variation of neutron concentration $n(t)$. Actual neutron measurements give us the following expression for the neutron count rate $N(t)=F(t) S$, where $S$ is the detector area. Thus, the mathematical approximation of the experimental count rate data:

$N(t)=n(t) v S$

will, in fact, be the mathematical approximation of neutron concentration in the neutron field of the Earth. Analysis of this approximation permits us to study properties of the neutron source and the nature of flux variations.

Earlier experiments (Kuzhevskij, 2000, 2001b), which included studies of the neutron flux in different geographic locations (on balloons: Apatity, Kolskij peninsula; Dolgoprudny, Moscow region; Ryl'sk, Kaluga region; groundbased experiments at different altitudes above sea level: Moscow; Golitsino, Moscow region; Seliger Lake, Tver' 
region; Pamir, Tadgikistan; Tyan-Shan, Kazakhstan) have shown that there seems to be at least two neutron sources near the Earth's crust. The first source is the generation of neutrons in the Earth's atmosphere as a result of interactions between high-energy space particles with nuclei of the different elements of the Earth's atmosphere. The second source consists of different radioactive gases (Radon, Thoron, Actinon), continuously released into the atmosphere by the Earth's crust. These gases produce $\alpha$-particles with energies ranging from 5 to $9 \mathrm{MeV}$. Nuclear interaction of $\alpha$ particles with nuclei of the elements of the Earth's crust and the atmosphere result in the production of neutrons. In other words, the Earth's crust itself is the second source of neutrons in the crust vicinity. Separation of this source of neutrons permitted us to show (Kuzhevskij et al., 2001b) that the observed short-period variations of the neutron flux are a result of the dynamic processes in the Earth's crust.

\section{Experimental technique}

The different physical principles and techniques used for processing the data recorded by the Detector of Nuclear Radiation of the Earth and its Atmosphere DAIZA (Russian abbreviation) are listed below:

Standard neutron counters SI-19N were used as detectors of the neutron component. These counters have the shape of a cylinder, with the length of $22 \mathrm{~cm}$ and diameter of $3.2 \mathrm{~cm}$. The sensitive area of the counter was $51 \mathrm{~cm}^{2}$. The counter was filled with ${ }^{3} \mathrm{He}$ gas pressurized to 4 atmospheres and operated in the coronal discharge mode.

The neutron capture reaction ${ }^{3} \mathrm{He}(\mathrm{n}, \mathrm{p}) \mathrm{T}$ with energy release of $760 \mathrm{keV}$ is the most efficient one. Stability tests of operation of neutron modules (containing 12 such counters), carried out under special conditions in the laboratory, showed that stability is not lower than $99 \%$ and changes slightly with neutron energy increase from thermal values to $2 \mathrm{MeV}$. In the range of neutron energies from thermal to $100 \mathrm{eV}$, the neutron detection efficiency for such counters is 0.8 . Currently, the neutron block consists of two planes, each with 40 counters. The two identical detectors used in the device permitted one to carry out research for different geometries and to study the energy distribution of neutrons using an absorbent (for instance, cadmium) and a retardant (for instance, plexiglass).

SBT-10 counters (with $\mathrm{E}_{e} \geq 45 \mathrm{keV}$ threshold for electrons, $\mathrm{E}_{p} \geq 0.5 \mathrm{MeV}$ for protons, $\mathrm{E}_{\alpha} \sim 5 \mathrm{MeV}$ for $\alpha$ particles ) and two Geiger-Muller counters STS-6 were used for detecting charged particles. One of the Geiger-Muller counters had a $0.5 \mathrm{~cm}$ thick aluminum cover. A scintillation crystal of $\mathrm{NaI}(\mathrm{Tl}) 7 \mathrm{~cm}$ long and $3 \mathrm{~cm}$ in diameter was used for detecting $\gamma$-radiation. There were two energy thresholds: $\mathrm{E}_{\gamma} \geq 300 \mathrm{keV}$ and $\mathrm{E}_{\gamma} \geq 2 \mathrm{MeV}$. In the present work the authors analyze mainly the data recorded by the neutron block of the instrument. Charged particle and $\gamma$-radiation data will be discussed in future papers.

\section{Analysis of the experimental results}

The DAIZA instrument has been continuously operated for several years with insignificant gaps, providing an extensive data base. For further analysis of neutron distributions we chose the data obtained during 1996, due to its homogeneity and completeness.

We obtained an approximation of the neutron count rate per second in both the direction towards the Earth's crust and away from it, using continuous measurements made in Moscow of neutrons at ground level. The neutron count rate can be described by the sum of two functions: a Poison distribution with parameters $a_{1}$ and $\gamma$, and a log-normal distribution with parameters $a_{2}$ and $b$ :

$f(m)=a_{1} \frac{\lambda^{m}}{m !} e^{-\lambda}+\frac{1}{a_{2} m} e^{-\left(\frac{\ln m-b}{a_{2}}\right)^{2}}$,

where $f(m)$ is the probability of observing a certain neutron count rate per second, and $m$ is the number corresponding to a certain neutron count rate per second.

The Poison distribution describes the count rate up to 5 neutrons per second (it corresponds to a neutron flux of $2.5 \times 10^{-3} \mathrm{~s}^{-1} \mathrm{~cm}^{-2}$ ), and the log-normal distribution describes the count rate over 5 neutrons per second. It is necessary to note that in fact a log-normal distribution was obtained for count rates up to $15 \mathrm{~s}^{-1}$; however, a count rate up to $50 \mathrm{~s}^{-1}$ can also be successfully described by this function. Although we sometimes observed significantly greater values of count rates $\left(100-200 \mathrm{~s}^{-1}\right)$, such values can hardly be used for approximation, due to the very low probability of their observation. For quiet periods (where count rates do not exceed $5 \mathrm{~s}^{-1}$ ), the complete approximation of the distributions is well described by the Poison distribution. The lognormal distribution is important in cases when count rates of the order of 10 or more neutrons per second are observed. Such a situation is unusual for quiet periods and occurs mainly during unquiet periods (the so-called "splashes"). Similar to our previous studies (Kuzhevskij et.al., 2001a), we selected unquiet periods. Unquiet periods are those for which hourly values of the neutron flux exceed the monthly-mean hour value by more than for $10 \%$. Studies of the seasonal dependence of the thermal neutrons count rate (Kuzhevskij et al., 2002a) have shown that neutron flux is anisotropic. The anisotropy value for quiet periods does not exceed $10 \%$, but its sign depends on the season. During the periods from January to March and from October to December, the anisotropy is positive; this means that during these periods the neutron flux moving towards the Earth prevails over the neutron flux in the direction away from the Earth. In June and July the anisotropy is negative, conforming to the prevalence of neutron flux coming away from the Earth. During the remaining months, the anisotropy is very low and it is impossible to affirm the prevalence of neutron flux from any direction.

Studies of the dependence of the splash period duration on the season show (Kuzhevskij et al., 2001a) that longer splash periods are more typical for the neutron flux, which 

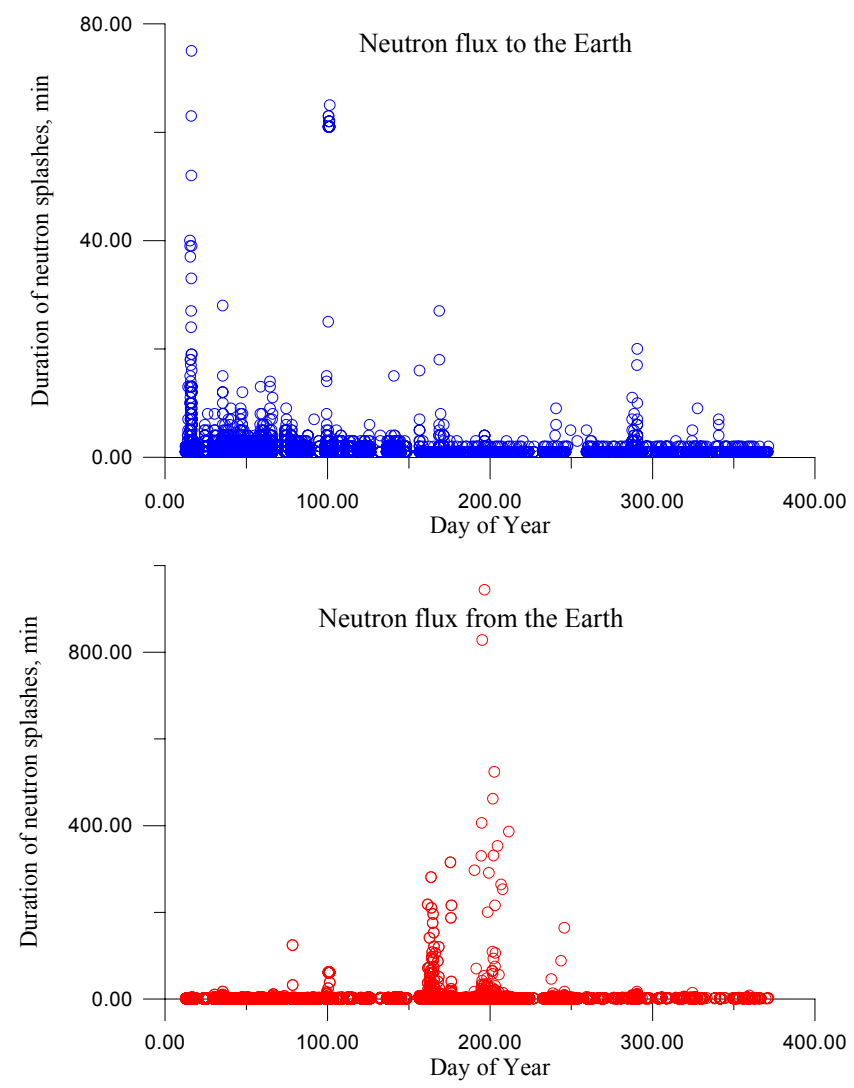

Fig. 1. Seasonal dependence of the duration for splash periods for neutron flux, directed to the Earth and from it. It is easy to see that for neutron flux directed from the Earth, the greatest number of splash periods occur in the middle of the year. For neutron flux, directed towards the Earth, the seasonal dependence is not so sharp and the duration of the splash periods is shorter, than for the opposite neutron flux direction.

propagates in the direction away from the Earth's crust, than for neutrons, propagating in the direction towards the Earth's crust (see Fig. 1). For neutron flux directed towards the Earth, splashes with maximum duration appeared in winter and in spring, and their duration did not exceed 60-80 min. For the neutron flux directed away from the Earth, the greatest number of long periods of splashes were observed during the summer, and they were much longer, up to 800 $1000 \mathrm{~min}$, or $14-16 \mathrm{~h}$. Hence, analysis of the neutron splash period duration leads to the conclusion that long splash periods are seasonally and directionally dependent.

The type of the approximation function (Eq. 3) confirms the conclusion (Kuzhevskij et al., 2002a; Volodichev et al., 2002) about the important contribution made by the Earth's crust to the total flux of thermal and slow neutrons in its vicinity. This conclusion was also obtained by another method (Kuzhevskij et al., 2001b; Volodichev et al., 2002) and it demonstrated the connection between neutron flux varitaions near the Earth's crust and its dynamics. This function confirms that this input of the Earth's crust comes through mainly during different types of splashes, which occur in the
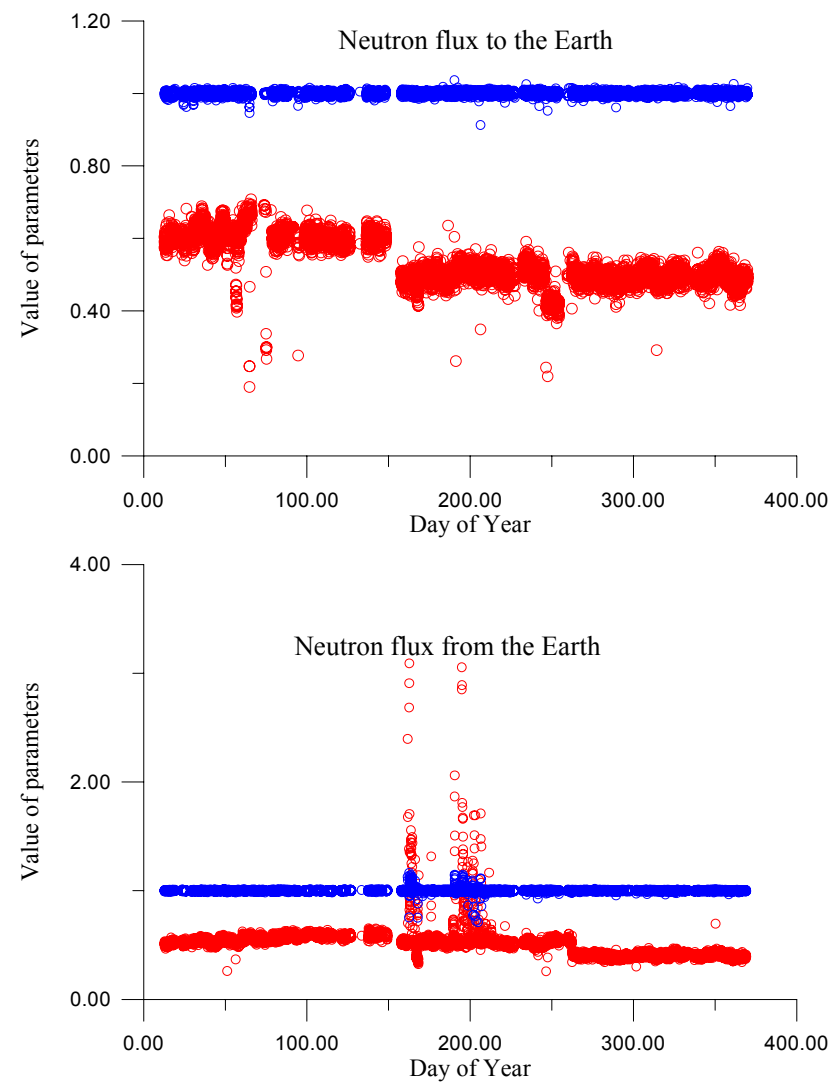

Fig. 2. Seasonal dependence of the approximation parameters $a_{1}$ and $\lambda$ of the Poison distribution, which describes the distribution of neutron flux, directed to the Earth and from it, during quiet periods. Blue points - parameter $a_{1}$, red points - parameter $\lambda$. The large number of points for the neutron flux, directed from the Earth, in the middle of the year (150-200 DOY) corresponds to the increasing of neutron fluxes from the Earth's surface during this period. As a result, the average counting rate for this period increases.

Earth's crust (splash periods).

Variations of the parameters in Eq. (3) were studied during 1996. Changes of Poison parameters $a_{1}$ and $\lambda$ are shown in Fig. 2 for quiet periods and in Fig. 3 for unquiet periods. These figures confirm that parameters of the Poison distribution for quiet periods do not change in terms of calculation accuracy for neutron fluxes directed either towards the Earth or away from it. Concerning the increase in the $\lambda$-parameter for the neutron flux directed away from the Earth, near the middle of the year (see Fig. 2, lower axes), it is necessary to note that it is connected with the total increase in the neutron flux away from the Earth during this period, which led to increasing values of the neutron flux averaged over one month, used for distinguishing between quiet and unquiet periods. Therefore, the neutron flux coming away from the Earth during quiet summer periods, which were selected according the above mentioned criterion, cannot be compared with the flux during quiet winter periods, because the mean monthly neutron fluxes directed away from the Earth differ greatly for the summer and winter periods. 

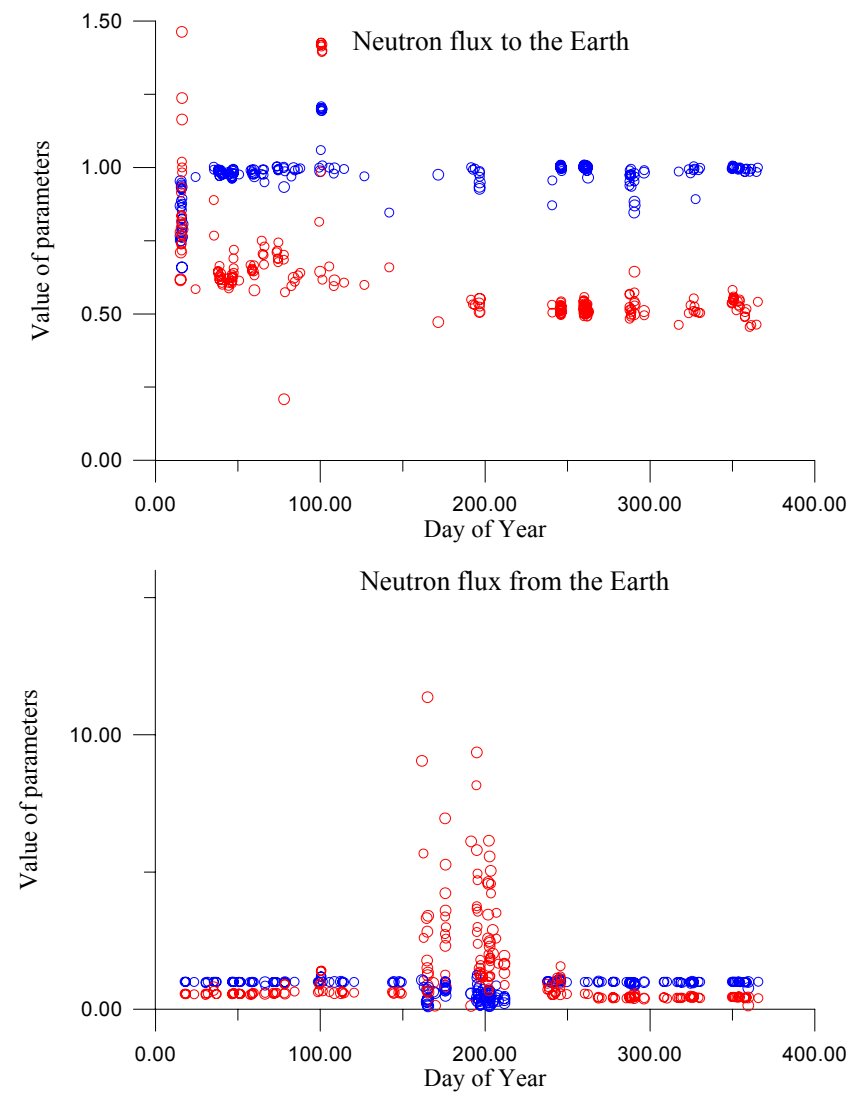

Fig. 3. The seasonal dependence of the approximation parameters $a_{1}$ and $\lambda$ of the Poison distribution, which describes the distribution of the neutron flux, directed towards the Earth and away from it, during unquiet periods. The blue points correspond to the $a_{1}$ parameter, the red points correspond to the $\lambda$ parameter. The large number of points for the neutron flux, directed away from the Earth, in the middle of the year (150-200 DOY) corresponds to the increase in neutron fluxes from the Earth's surface during this period.

Taking into account unquiet periods of registration (see Fig. 3), we can add that in most cases for the neutron fluxes directed both towards the Earth's surface and away from it, the Poison parameters of distribution do not change greatly except during the summer period for the neutron flux directed away from the Earth's surface, which we have already discussed. This means that a large part of these cases are regarded as unquiet periods, due to the recording of a large number of neutrons (over 5 neutrons per second), but not due to the increase in the probability for a small number of neutrons (up to 5 neutrons per second), since the Poison function describes the very beginning of a distribution up to 5 neutrons per second.

Figure 4 presents the seasonal dependence of log-normal parameters $a_{2}$ and $b$. As we have mentioned above, this part of the distribution is important only for unquiet periods, when the count rate exceeds 5 neutrons per second. Again, a large amount of points in the middle of the year for the neutron flux directed away from the Earth indicates a total increase in the neutron flux during this period and a totally
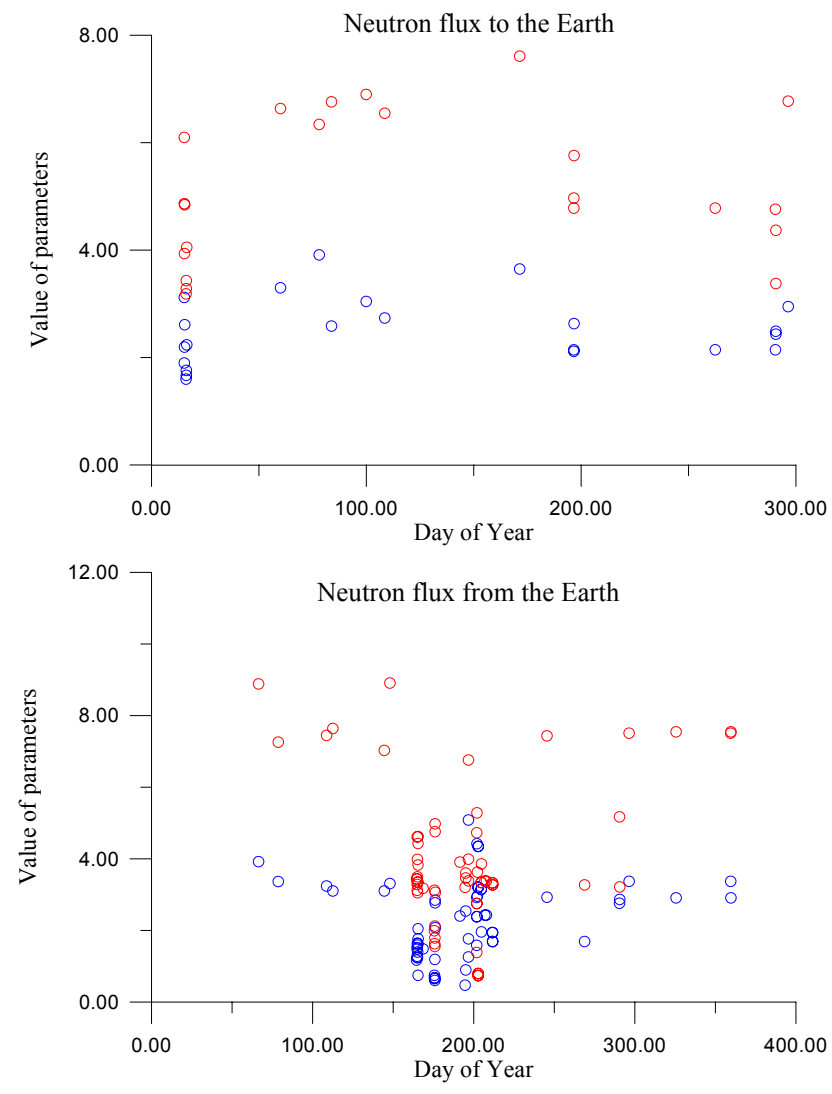

Fig. 4. Seasonal dependence for the approximation parameters $a_{2}$ and $b$ (according to Eq. 3) of the log-normal distribution, which describes the distribution of the neutron flux, directed towards the Earth and away from it, during unquiet periods. The blue points represent parameter $a_{2}$, the red points represent parameter $b$. The large number of points for the neutron flux, directed away from the Earth, in the middle of the year (150-200 DOY) corresponds to an increase in neutron fluxes from the Earth's surface during this period.

"unquiet" situation in the Earth's crust.

Figure 5 shows separate cases of the full approximation of neutron count rates, for propagation towards the Earth's surface and away from it, during splash periods. Figures 6-8 present a very interesting event which occurred on 13 July 1996. Here we can see in consecutive order changes in the probability distribution for the neutron flux, directed away from the Earth. It is necessary to note that for the flux directed towards the Earth during the same period of time, nothing extraordinary was observed: for this direction this period is quiet, so the probability distribution is short and is well approximated by a single Poison function. The neutron count rate exceeds the mean-monthly value up to 50 times (see Fig. 6) for several hours. This gives us an opportunity to study time variations of the probability distribution and the distribution parameters for this period. Changes in the neutron probability distributions are shown in Fig. 7, where we can see the appearance, growth and slump of the disturbance. All these distributions are approximated with the sum of the 

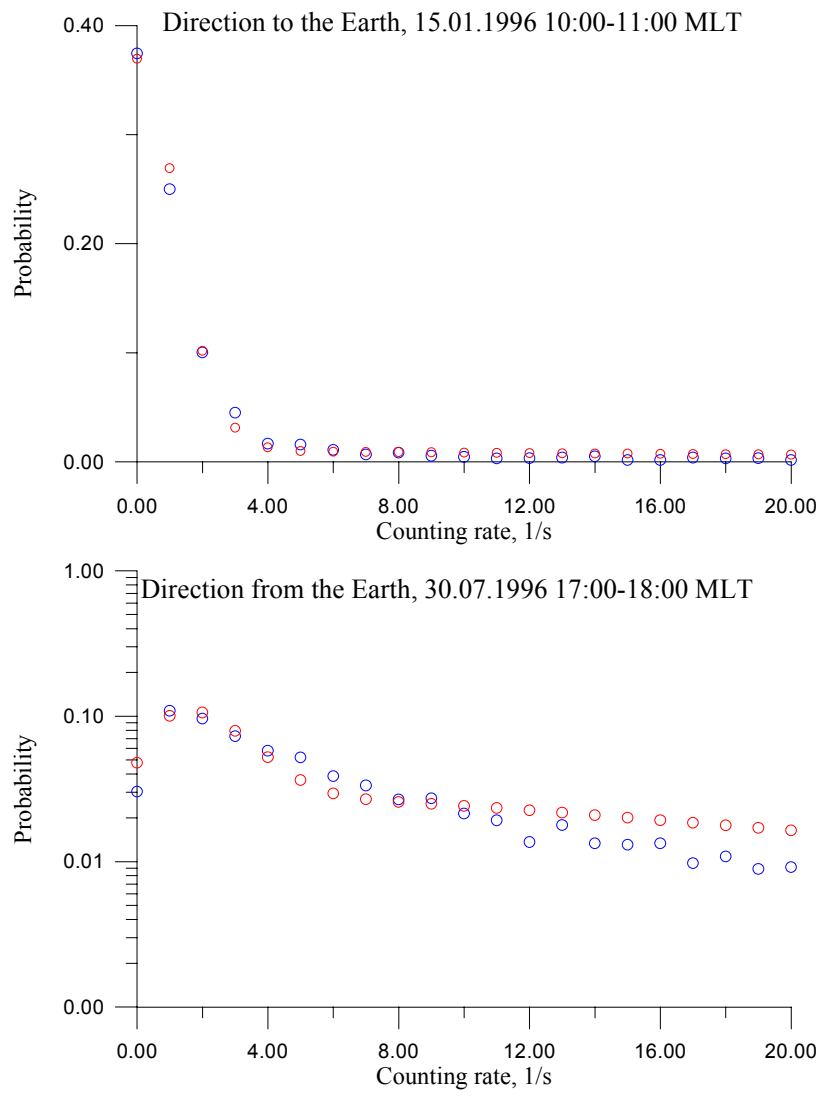

Fig. 5. Examples of approximations for the neutron flux probability distribution in the directions towards the Earth and away from it, using the sum of a Poison distribution and a log-normal distribution. The blue points are experimental data, the red points are the approximation function. For the upper axes the approximation parameters (according to Eq. 3 ) are the following: $a_{1}=0.751, \lambda=0.709$, $a_{2}=2.609, b=4.842$. For the lower axes the approximation parameters (according to Eq. 3 ) are the following: $a_{1}=0.327$, $\lambda=1.941, a_{2}=1.685, b=3.265$.

Poison and log-normal functions:

$f(m)=a_{1} \frac{\lambda^{m}}{m !} e^{-\lambda}+a_{2}+b e^{-0.5\left(\frac{i \mathrm{n}(m / c)}{d}\right)^{2}}$.

In fact, this is Eq. (3), but written in a simplified form, because the log-normal distribution includes the variable $m$ only as an argument of the exponential function. This form of the log-normal distribution is used in the "TableCurve $\mathrm{e}^{\mathrm{TM}}$ for Windows" code, which we used to approximate the distributions.

Figure 8 presents movement of the point of maximum probability of registration for the "tail" of distribution.

\section{Discussion}

Study of the neutron count rate distribution near the Earth's crust is of essential importance in several aspects. On the one hand, it allows one to separate different neutron sources

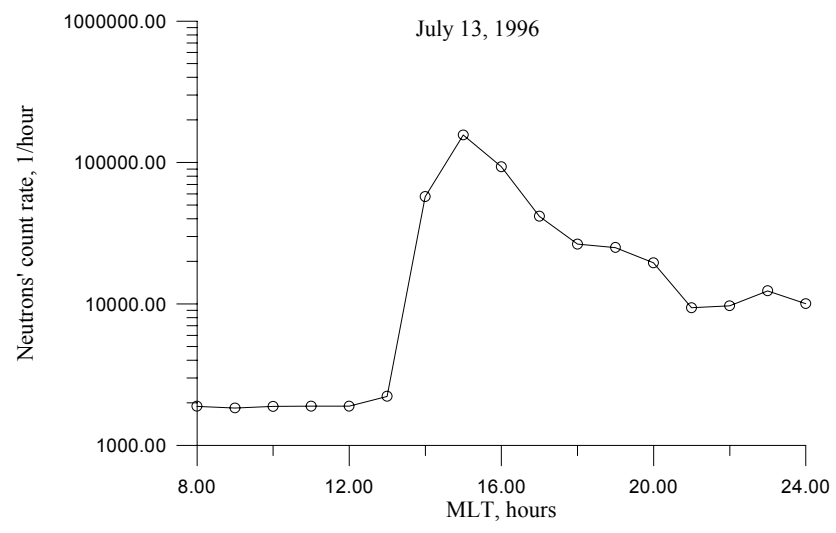

Fig. 6. Hourly neutrons count rate for neutrons, directed away from the Earth's surface, for a long disturbed period on 13 July 1996.

(space sources, including solar flares (Kuzhevskij et al., $2002 b$ ) and sources of terrestrial origin), which produce neutron fluxes in the lower atmosphere. On the other hand, it provides new information about the nature of neutron flux variations in the lower atmosphere and its connection with dynamics of the neutron source. Besides, this approach permits one to study dynamic processes in the source, using neutron flux variations. Moreover, studies of the neutron count rate distribution dynamics near the Earth's crust give the opportunity to use a new approach to the solution of a very important scientific problem - short-term prediction of earthquakes and volcano activity (Kuzhevskij, 2000).

Taking into consideration all of the obtained results. we can assert that for quiet periods of registration, the probability distribution includes count rates of up to 5 neutrons per second. These distributions can be approximated by a Poison function with a high degree of accuracy. For unquiet periods the probability distribution can include count rates of several dozen and even hundreds of neutrons per second. In order to approximate such distributions, it is necessary to use the sum of the Poison function and log-normal function.

It is necessary to note the main difference between the probability distributions for the neutron flux propagating towards the Earth and away from it. While during the unusual periods of recording the neutron flux directed towards the Earth, with maximum probability, always corresponds to the count rate of zero neutrons per second, the neutron flux directed away from the Earth, with maximum probability, can shift to count rates of 1-2 neutrons per second, and sometimes can even reach 5 neutrons per second.

Although the probability distributions can be approximated with the sum of these functions up to neutron count rates of 15 neutrons per second for neutrons coming from both directions (towards the Earth and away from it), the probability of such values for neutron flux directed towards the Earth is very small. Therefore, the reliability of such an approximation is low, while the neutron flux coming away from the Earth is approximated, using the above mentioned function, with the accuracy of about $95 \%$. This conclusion is 


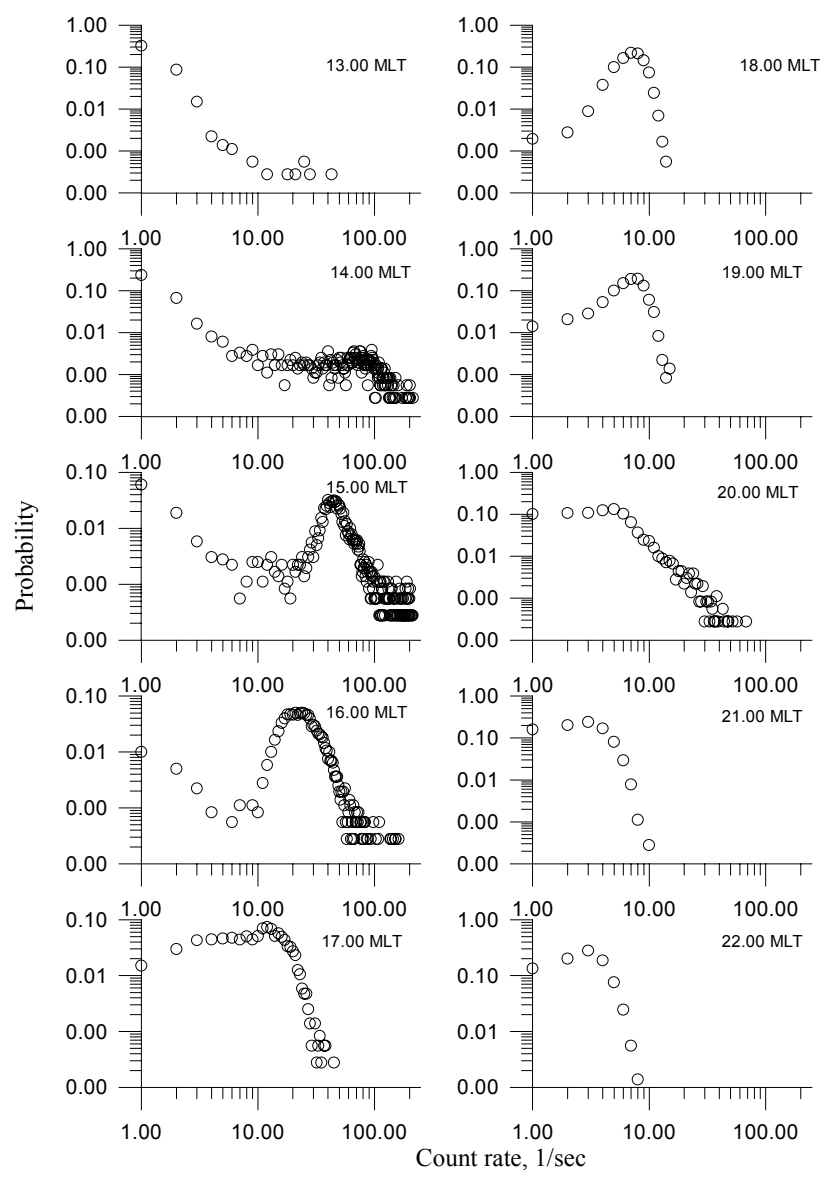

Fig. 7. Consecutive change in the probability distribution for the neutron flux, directed away from the Earth, for a long disturbed period on 13 July 1996 . Each axis presents the probability distribution for a 1-h sub-period of registration.

based on thorough studies of all unquiet periods of recording for both neutron fluxes directed towards the Earth and away from the Earth. Analysis of the approximation parameter changes during the whole year has shown that there is no seasonal dependence: the Poison parameters remain stable, with some increases during the whole year. The only case that contradicts this conclusion contains summer observations of the neutron flux directed away from the Earth. This period is characterized by strong variations of distribution parameters, which are caused by strong and sudden changes in the neutron flux, observed during this period. This fact confirms our earlier statement that during the warm and dry season, when the Earth's surface is not covered with any absorber, neutrons originating from radioactive gas exhaust from the Earth's crust can be easily detected.

We suppose that the input of two independent sources of thermal neutrons near the Earth's surface into the probability distribution of neutron flux can be described by the sum of two distributions. The first source is connected with the generation of neutrons during interactions between high-energy space particles with nuclei of the Earth's atmosphere elements. Its input into the distribution of the neutron flux can

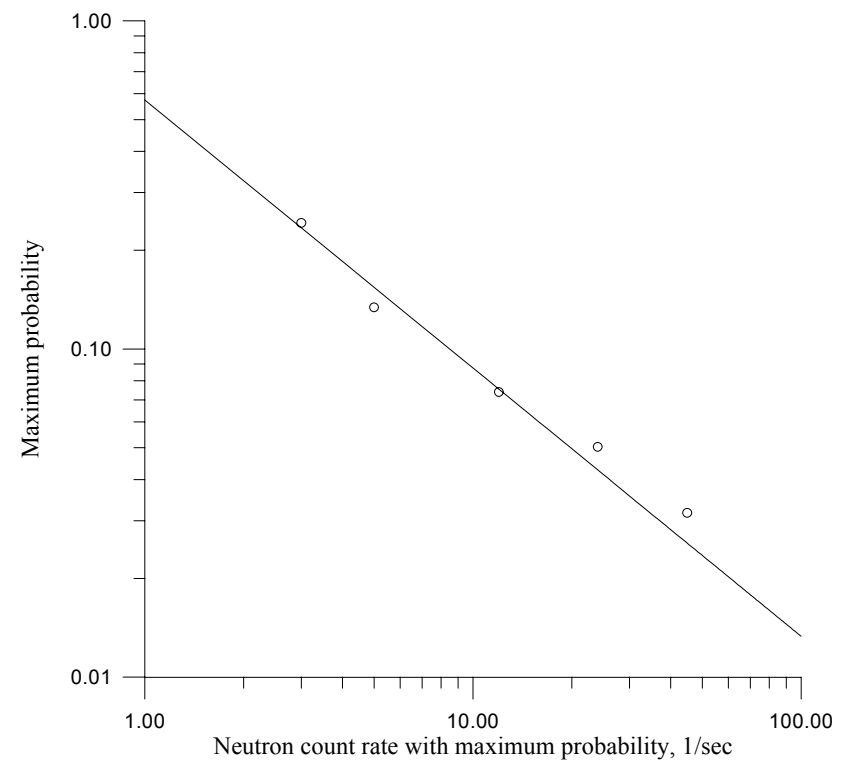

Fig. 8. Time dependence for the point of maximum recording probability for the "tail" of the distribution on 13 July 1996. The experimental data points are approximated with the function $\ln \mathrm{P}\left(\mathrm{N}_{\max }\right)=$ $\mathrm{a}+\mathrm{blnN}_{\max }$ with $a=-0.5555, b=-0.8171$.

be described by a Poison function with parameters $a_{1}$ and $\lambda$. This function describes accurately the probability distribution of neutron fluxes moving in both directions, away from the Earth and towards it, during the quiet periods of registration, when these parameters change weakly. It is necessary to note that the total duration of quiet periods is about $90 \%$ of the total database for 1996. Most of the unquiet periods parameters of the Poison distribution also change weakly. This means that the source of neutrons, which is described by this function, is stable, and there are seldom variations in its contribution to the neutron flux near the Earth's crust.

The second source of neutrons near the Earth's crust is connected with the release of radioactive gases, generating $\alpha$-particles, and further production of neutrons during interactions between $\alpha$-particles and nuclei of the elements of the Earth's crust and the atmosphere. The contribution of this source to the distribution of neutron flux near the Earth's crust can be described by a log-normal distribution with parameters $a_{2}$ and $b$. As we have discovered, this distribution becomes important for the neutron flux directed away from the Earth during unquiet periods.

The influence of the Earth's crust as a neutron source can have a different duration and a different character. It can be a pulsed source, or a long-term source, with monotonous or non-monotonous temporal behavior, for instance, multipulsed source. In the case of a pulsed source, the neutron flux variation can be described by a temporal diffusion profile, because the increased flux duration will depend only on neutron scattering and absorption processes. According to the lifetime of neutrons with a primary energy of about 1$2 \mathrm{MeV}$ near the Earth's surface (Beliaeva et al., 1999), the duration of such an event will be short, about several seconds. 
In the case of a monotonously decreasing long-term source, the temporal profile of the neutron flux will also be diffusionlike, but the slump will be longer, and the parameters, describing the time variation, will change monotonously. These parameters include approximation parameters of the neutrons' count rate distribution. The neutron splash of 13 July 1996 is an example of such an event.

Certainly, the event of 13 July 1996 is one of the most interesting subjects for studies. The proposed function successfully describes even complicated cases of such "longtailed" distributions (up to the end), although the distribution parameters change greatly. The neutron flux returned to the "background" value of the evening of 14 July 1996. We studied only a part of this long splash period, where temporal connections between the maximum value of probability and the corresponding neutron count rate, change monotonously. The possibility of applying this function in such cases once again confirms the correctness of our approach. There are several similar summer periods for the neutron flux, directed away from the Earth, during which the probability distribution has an unusual peak in the area of high count rates (its value can change from dozens to hundreds of neutrons per second). Studying the dynamics of such disturbances we find that this peak (see Fig. 8) shifts from high to low count rates with a simultaneous increase in probability value. This means that the impact of the source decreases.

In the case of a multi-pulsed source, the parameters of the distribution function will change non-monotonously. Such events were also observed. For instance, during the event of 15 July 1996, we observed a monotonous change in the count rate value, which corresponds to the maximum probability value, and then for several hours the picture remained stable, and the count rate value for maximum probability value did not change. This could mean are additional production of neutrons in the source.

For such prolonged disturbed periods it is even possible to study the change in approximation parameters both for the Poison and log-normal distributions. It is known that dynamic processes in the Earth's crust can be caused by several reasons. Earthquakes and increasing volcano activity are two of the most powerful sources. Without any doubt these phenomena are not sudden. Preceeding them there is a certain period of energy accumulation and increase in the Earth's crust deformation. As it follows from all the results mentioned above, we can conclude that this period should be observable in the variations of neutron flux coming away from the Earth's crust.

\section{Conclusions}

Studies of the neutron count rate distribution in the neutron field of the Earth for two vertical directions, towards the Earth and away from it, show that:

1. A complete approximation of this distribution can be presented as a sum of two functions - a Poison distribution and a log-normal distribution. The maximums of the neutron counting rate distributions for different directions are different. For neutrons, moving towards the Earth's crust, the maximum of the probability distribution is located near the count rate of 1-5 neutrons per second, for the opposite direction - the maximum of the probability distribution is near the zero count rate.

2. The log-normal distribution is important for splash periods which are characterized by neutron count rates of 10 and more neutrons per second. For these periods the probability values of count rates over 10 neutrons per second for neutron flux directed away from the Earth are 10 times greater than the corresponding probability values for the same count rates of the neutron fluxes towards the Earth. Splash periods are connected with dynamical processes in the Earth's crust; consequently, log-normal distribution describes the Earth's crust as a source of neutrons.

3. In the case of long disturbed periods (with duration of over several hours), maximum probability changes from hour to hour. The value of maximum probability increases while the value of the corresponding count rate decreases. Mathematically the connection between the values of maximum probability and the corresponding count rate can be described by the expression $\ln \mathrm{P}\left(\mathrm{N}_{\max }\right)=\mathrm{a}+\operatorname{bln} \mathrm{N}_{\max }$, where $\mathrm{P}\left(\mathrm{N}_{\max }\right)$, is the maximum probability and $\mathrm{N}_{\max }$ is the corresponding neutron count rate. Breaches of this connection can be explained in terms of concepts, which describe the Earth's crust as a source of neutrons in the lower atmosphere (they are discussed in our publications). This can occur due to repeated dynamical impacts on the Earth crust and, consequently, due to repeated processes of neutron generation.

4. Detailed studies of neutron flux distribution parameter variations near the Earth's crust can serve as the basis for developing a nuclear-physics technique for shortterm prediction of natural hazards.

Acknowledgements. We are very grateful to editorial office and referees for detailed remarks and to E. D. Tolstaya for her help in article preparence.

\section{References}

Beliaeva, E. A., Kuzhevskij, B. M., Nechaev, O. Yu., and Panasyuk, M. I.: Neutron sources and nature of time variations of neutron flux near the Earth surface, Physical Ecology, Moscow, 4, 89-96, 1999.

Gorshkov, G. V., Zabkin, V. A., Lyatkovskaya, N. M., and Tsvetkov, O. S.: Natural neutron background of the atmosphere and the Earth's crust, M., Atomizdat, 82-117, 1966.

Kuzhevskij, B. M., Nechaev, O. Yu., and Shavrin, P. I.: Anisotropy of thermal neutrons in the Earth's atmosphere, Geomagnetism and Aeronomy, 35, 2, 166-168, 1995. 
Kuzhevskij, B. M., Nechaev, O. Yu., Shavrin, P. I., Beliaeva, E. A., and Sirotkin, I. I.: Variations of neutron concentration in thermalneutron field of the Earth's atmosphere, Preprint SINP MSU 967/414, 2-3, 1996.

Kuzhevskij, B. M.: Nucleonics-based monitoring of the ecological environment balance of the Earth crust and lower atmosphere, http://www.srd.sinp.msu.ru/bmk/nuclear.doc, 2000.

Kuzhevskij, B. M., Nechaev, O. Yu., Panasyuk, N. I., and Sigaeva, E. A.: Seasonal dependence of thermal neutrons anisotropy near the Earth surface (unquiet periods) Physical Ecology, Moscow,7, 101-109, 2001a.

Kuzhevskij, B. M., Nechaev, O. Yu., Panasyuk, M. I., Sigaeva, E. A., Volodichev, N. N., and Zakharov, V. A.: Neutron field of the
Earth, Origin and Dynamics, Journal of the Korean Association for Radiation Protection, 26, 3, 315-319, $2001 \mathrm{~b}$.

Kuzhevskij, B. M., Nechaev, O. Yu., and Sigaeva, E. A.: Seasonal dependence of thermal neutrons anisotropy near the Earth surface, Vestnik MSU, Ser. Phys. and Astr., 1, 55-58, 2002a.

Kuzhevskij, B. M., Nechaev, O. Yu., and Sigaeva E. A.: A new method of registration of neutron from solar flare, Proc. of Conf. of CIS and Baltic states, SPb., 318-320, 2002b.

Yuan, L. C. L.: Altitudinal dependence of slow neutrons' flux by baloon experiments, Bull. Amer. Phys. Soc., 23, 2, 21-25, 1948.

Volodichev, N. N., Zakharov, V. A., Kuzhevskij, B. M., Nechaev, O. Yu., and Sigaeva, E. A.: The Earth crust is an active neutron source, Vestnik MSU, Ser. Phys. and Astr., 5, 69-73, 2002. 